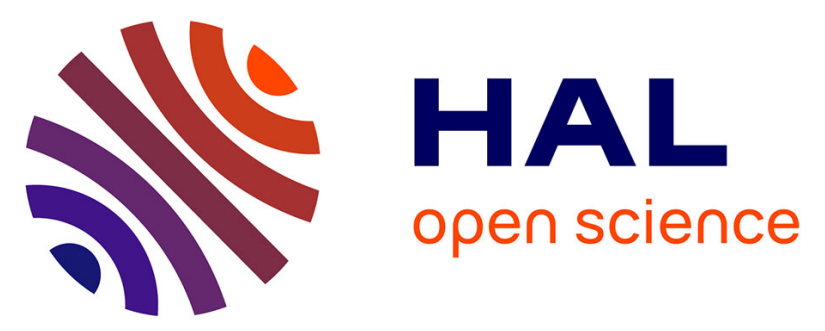

\title{
Molecular orbital tomography from multi-channel harmonic emission in N2
}

\author{
Z Diveki, R Guichard, J Caillat, A Camper, Stefan Haessler, T Auguste, T \\ Ruchon, B Carré, A Maquet, R Taïeb, et al.
}

\section{- To cite this version:}

Z Diveki, R Guichard, J Caillat, A Camper, Stefan Haessler, et al.. Molecular orbital tomography from multi-channel harmonic emission in N2. Chemical Physics, 2013, 414, pp.121. 10.1016/j.chemphys.2012.03.021 . hal-01164764

\section{HAL Id: hal-01164764 \\ https://hal-ensta-paris.archives-ouvertes.fr/hal-01164764}

Submitted on 17 Jun 2015

HAL is a multi-disciplinary open access archive for the deposit and dissemination of scientific research documents, whether they are published or not. The documents may come from teaching and research institutions in France or abroad, or from public or private research centers.
L'archive ouverte pluridisciplinaire HAL, est destinée au dépôt et à la diffusion de documents scientifiques de niveau recherche, publiés ou non, émanant des établissements d'enseignement et de recherche français ou étrangers, des laboratoires publics ou privés. 


\title{
Molecular Orbital Tomography from Multi-Channel Harmonic Emission in $\mathrm{N}_{2}$
}

\author{
Z. Diveki ${ }^{1}$, R. Guichard ${ }^{2}$, J. Caillat ${ }^{2}$, A. Camper ${ }^{1}$, S. Haessler ${ }^{3}$, T. Auguste ${ }^{1}$, T. Ruchon ${ }^{1}$, B. Carré $^{1}$, A. Maquet ${ }^{2}$, R. \\ Taïeb $^{2}$ and P. Salières ${ }^{1}$ \\ ${ }^{1}$ CEA-Saclay, IRAMIS, Service des Photons, Atomes et Molécules, 91191 Gif-sur-Yvette, France \\ ${ }^{2}$ UPMC Université Paris 6, UMR 7614, Laboratoire de Chimie Physique-Matière et Rayonnement, 11 rue Pierre et Marie Curie, 75231 Paris \\ Cedex 05, France, \\ CNRS, UMR 7614, LCPMR, Paris, France \\ ${ }^{3}$ Photonics Institute, Vienna University of Technology, Gußhausstraße 27/387, 1040, Vienna, Austria
}

\begin{abstract}
High-order harmonic generation in aligned molecules can be used as an ultrafast probe of molecular structure and dynamics. By characterizing the emitted signal, one can retrieve information about electronic and nuclear dynamics occurring in the molecule at the attosecond timescale. In this paper, we discuss the theoretical and experimental aspects of molecular orbital tomography in $\mathrm{N}_{2}$ and investigate the influence of multi-channel ionization on the orbital imaging. By analyzing the spectral phase of the harmonic emission as a function of the driving laser intensity, we address two distinct cases, which in principle allow the orbital reconstruction. First, the contributions from two molecular orbitals could be disentangled in the real and imaginary parts of the measured dipole, making it possible to reconstruct both orbitals. Second, by decreasing the driving laser intensity, the transition from a multi-channel to a single-channel ionization regime is shown. The highest occupied molecular orbital may then be selected as the only one contributing efficiently to the harmonic emission. The latter approach paves the way towards the generalization of tomography to more complex systems.
\end{abstract}

Keywords: High-order harmonic generation, Strong field physics, Molecules

\section{Introduction}

The fast development in laser technology provided a tool to study the rearrangements of molecular nuclei during a chemical reaction on the femtosecond $(1 \mathrm{fs}=$ $10^{-15} \mathrm{~s}$ ) timescale [1]. The next challenge is to observe charge rearrangements occurring in the molecular structure after photoexcitation. The molecular energy difference between electronic states can reach up to several (tens of) eVs, leading to dynamic processes taking place on the attosecond $\left(1\right.$ as $\left.=10^{-18} \mathrm{~s}\right)$ timescale and spatially confined to sub-nanometer scaled volumes [2]. Achieving such spatio-temporal resolution would allow investigating many fundamental processes in physics, chemistry and biology and most importantly, would provide a route to control them.

Attosecond pulses are now routinely produced using a process called High-order Harmonic Generation (HHG) $[3,4]$. This highly non-linear interaction occurs when intense infrared (IR) laser pulses are focused in atomic or molecular gas jets. This process can be described by a succession of three steps $[5,6,7]$ where:

Preprint submitted to Chemical Physics first, the strong laser field creates a Coulombic barrier through which an electron can tunnel in the continuum within a fraction of one optical half-cycle (step i), thus creating a coherent superposition of bound- and continuum electronic wave-packets (EWP). Second, the continuum EWP is accelerated away and then back to the ionic core (step ii). Finally, the recollision of the EWP with the core may result in recombination with emission of an attosecond burst of XUV photons (step iii). For a multi-cycle laser pulse, this process is repeated every half-cycle resulting in the emission of a train of attosecond pulses, the spectrum of which is composed of odd harmonics of the fundamental laser frequency. These isolated- or trains of- attosecond pulses can now be precisely characterized temporally $[8,9,10,11]$.

Their applications to attosecond spectroscopy follow two different directions. The first one relies on a well characterized harmonic source and exploits a conventional pump-probe scheme where the generated attosecond pulses are used to excite or probe ultrafast dynamics in a target system $[12,14,15,16,17]$. In the second one, 
called self-probing scheme, harmonics are generated directly in the target system and the information carried by the attosecond emission is recovered by precisely characterizing it. It exploits the fact that the re-colliding EWP "probes" the ionic core, with Ångström de Broglie wavelength, attosecond duration and sub-driving laser cycle excursion time. The molecular structure and dynamics become accessible on their own natural dimension with unprecedented temporal resolution. More precisely, these structural and dynamical informations are encoded in the recombination dipole moment, i.e. in the amplitude, phase, polarization (e.g. ellipticity) of the harmonic emission. One thus gets access to static parameters such as internuclear separation [18, 19, 20, 21], or orbital symmetry $[22,25,26]$ but also to rotational[27, 24], vibrational- [28, 29, 31, 32] and electronic$[33,34]$ dynamics occurring in the molecule. The attosecond emission is also sensitive to correlated multielectron effects, such as intra-ionic transitions during the EWP recombination [35]. The combination of the self probing scheme with an additional pumping laser pulse inducing for instance a photo-dissociation, allows resolving the temporal evolution of an ultrafast chemical reaction [36, 37].

In this article we focus on a very specific implementation of the self-probing scheme called molecular orbital tomography, first proposed by Itatani et al. [38]. It allows for the reconstruction of the amplitude and phase of the molecular orbital involved in the emission process from the advanced characterization of the harmonic emission. This promising technique "measures" the valence orbitals of complex molecules, and most importantly bears the potential of following in real time their distortion during an excitation, e.g., chemical reactions, on an attosecond timescale. However, several theoretical and experimental challenges must be overcome before reaching this goal. One of them is raised by the simultaneous contribution of multiple ionization channels to HHG [33, 39, 40]. It results in a harmonic emission built from the coherent sum of multiple contributions that drastically complicates the retrieval of information on individual orbitals. By reviewing the results of Haessler et al. [34] and Diveki et al. [41] obtained in $\mathrm{N}_{2}$ and supporting them with additional measurements, we show that, in specific experimental conditions, the existence of multi-orbital contributions does not prohibit the application of molecular orbital tomography. It is then even possible to reconstruct the wavefunctions of the two Highest Occupied Molecular Orbitals (HOMO and HOMO-1) contributing to HHG. In the general case, we propose a method allowing us to identify multi-orbital contributions. By decreasing the driving laser intensity, we then show that the contributions of the lower lying orbitals become negligible. The generalization of this approach may simplify the reconstruction of single radiating orbitals.

The article is organized as follows. In Section 2, we shortly describe the theoretical methods used throughout this work. The basic principles of molecular orbital tomography are reviewed and we discuss the possible theoretical and experimental obstacles together with the possibilities of improvement. The experimental methods are detailed in Section 3 and applied to the case of $\mathrm{N}_{2}$. The results are presented and discussed in Section 4. We first investigate the separation of the different orbital contributions (Section 4.1) and perform orbital tomographic reconstruction. We then study the possibility of selecting a single contribution (Section 4.2). We compare the laser intensity dependence of the calibrated intensity and phase of the harmonic emission and show that the latter is a very sensitive probe of multiorbital contributions. Finally, we conclude in Section 5.

\section{Theoretical Models}

\subsection{Principles of the Self-Probing Scheme}

Molecular orbital tomography has its origins in the self-probing scheme which is derived from the quantum description of HHG. The harmonic field is determined by the Fourier transform of the acceleration of the induced molecular dipole. Through the Ehrenfest theorem, the harmonic signal can be expressed in three different dipole forms $(f)$ : acceleration $(\hat{\mathbf{a}})$, velocity $(\hat{\mathbf{v}})$, length $(\hat{\mathbf{r}})$ [43]. In principle, all forms are equivalent, however due to various approximations defined hereafter, they may not give the same result $[44,45]$. It is assumed that the laser does not induce multi-electron effects (single active electron approximation, SAE) and that the influence of the ionic core on the freed EWP is negligible with respect to the strong laser field (strong field approximation, SFA). Using these approximations, and restricting to the contribution of the shortest possible EWP trajectories [42], the harmonic dipole can be factorized into three main terms: the tunnel ionization amplitude $(\gamma)$, the continuum acceleration amplitude (a), describing the accumulated phase and wave-packet spreading, and the recombination (d) [7, 46, 47, 48, 49]. The choice of the dipole form affects only the recombination dipole moment (RDM). The harmonic dipole then writes:

$$
\mathbf{D}\left(\omega, I_{L}, \theta\right)=\eta_{f} \gamma\left(\mathbf{k}, I_{L}, \theta\right) a\left(\mathbf{k}, I_{L}\right) \mathbf{d}^{f}(\mathbf{k}, \theta)
$$


where $\eta_{f}$ is a pre-factor depending on the dipole form, $\mathbf{d}^{f}(\mathbf{k}, \theta)=\left\langle\psi_{0}(\theta)\left|\hat{\mathbf{d}}^{f}\right| \psi_{\mathrm{c}}(\mathbf{k})\right\rangle$ is the complex RDM vector in the $f$ form with $\psi_{0}$ and $\psi_{c}$ being the ground and continuum states of the electron respectively, $\theta$ is the angle between the main axis of the molecule and the polarization of the driving laser field, $I_{L}$ is the intensity of the driving laser, $\omega$ is the angular frequency of the emitted harmonic and $\mathbf{k}$ is the asymptotic wavevector of the continuum electron. The energy conservation law connects the wavenumber of the EWP with $\omega$ through (in a.u.):

$$
\omega=\frac{k^{2}}{2}+I_{\mathrm{p}}
$$

where $I_{p}$ is the ionization potential. The direction of $\mathbf{k}$ is assumed parallel to the driving laser polarization that corresponds to the $\mathrm{z}$ direction in the laboratory frame, where $y$ represents the propagation direction of the generating laser beam, $x$ being perpendicular to both directions. The molecular frame, defined by the main axis of the molecule, $z^{\prime}$, and its secondary axis x', corresponds to a rotation of angle $\theta$ of the laboratory frame around the axis $y\left(=y^{\prime}\right)$.

Most of the information on the molecular structure and dynamics is encoded in the RDM. The latter can be retrieved from fully characterized HHG if one can calibrate for the first three terms in Eq. 1. Such a calibration could be performed using HHG from an atomic gas with same ionization potential under the same experimental conditions, provided that its RDM can be calculated or is known from other experiments [52]. Indeed, within the SFA, the factor $a\left(\mathbf{k}, I_{L}\right)$ is determined by the laser intensity $I_{L}$ and is considered independent of the target, whether it is an atom or a molecule, if they have the same $I_{p}$. In the case of molecules [50], the tunneling ionization probability $\gamma$ contains two factors: the first one is mainly described by the exponential behavior of tunnel ionization (Keldysh-theory, [51]), and is considered to be the same for atoms and molecules if $I_{p}$ and $I_{L}$ are identical. The molecular orbital geometry is accounted for by the second factor $R(\theta)$ which also depends on the laser intensity. Therefore, from the first three terms in Eq. 1, only $R(\theta)$ remains after calibration of the molecular signal by the reference atom emission. $R(\theta)$ has to be retrieved either theoretically or by measurements $[50,53,54,55]$. Note that the calibration also gets rid of $\eta_{f}$ and eliminates the problem of characterizing the spectral response of the detector. However, reference atoms may introduce some difficulties: any structural features in their response, like Cooper minima or resonances [35], may spoil the orbital reconstruction if the reference RDM is not precisely calculated. Moreover, reference atoms do not exist for all molecules, hence theoretically constructed references may be needed.

With the described calibration, the self-probing scheme allows us to access the complex RDM vector:

$$
\mathbf{d}^{f}(\mathbf{k}, \theta)=\frac{1}{R(\theta)} \frac{\mathbf{D}\left(\omega, I_{L}, \theta\right)}{D_{\mathrm{ref}}\left(\omega, I_{L}\right)} d_{\mathrm{ref}}^{f}(\mathbf{k}),
$$

where $d_{\text {ref }}^{f}$ is the reference atom RDM and $D_{\text {ref }}\left(\omega, I_{L}\right)$ is the corresponding measured harmonic signal, which are scalar quantities. Equation 3 implies that the complete characterization (in amplitude and phase for both vector components) of the harmonic emission from a molecule and from a reference atom gives direct access to the complex recombination dipole moment in the molecular frame.

\subsection{Principles of Molecular Orbital Tomography}

Itatani et al. [38] proposed to go one step further in the analysis of the RDM. Using the plane wave approximation (PWA) for the continuum states $|k\rangle\left(\psi_{\mathrm{c}}(\mathbf{k})=\right.$ $\left.\exp \left(\mathbf{i k} \cdot \mathbf{r}^{\prime}\right)\right)$, the molecular orbital itself can be expressed from the RDM by performing an inverse spatial Fourier transform. The different aspects of molecular orbital tomography are detailed in [43].

Since no induced dipole exists in the laser propagation direction, the contribution of the molecular orbital to the RDM is defined as the 2 dimensional projection onto the plane perpendicular to the propagation of the laser: $\tilde{\psi}_{0}\left(x^{\prime}, z^{\prime}\right)=\int \psi_{0}\left(x^{\prime}, y^{\prime}, z^{\prime}\right) \mathrm{d} y^{\prime}$. Consequently, an orbital with an odd symmetry in y' will not contribute to the dipole. Now we derive the tomographic orbital reconstruction in the length form where the dipole operator is $\hat{\mathbf{d}}^{\hat{\mathbf{r}}}=\mathbf{r}^{\prime}$ and we assume that the molecular orbital is real valued. Then the $u$-component $\left(u=x^{\prime}, z^{\prime}\right)$ of the dipole matrix element in the molecular frame writes:

$$
\begin{aligned}
d_{u}^{\hat{\mathbf{r}}}(\mathbf{k}) & =\left\langle\psi_{0}|u| k\right\rangle \\
& =\iint u \psi_{0}\left(x^{\prime}, z^{\prime}\right) \mathrm{e}^{\mathrm{i}\left(k_{x^{\prime}} x^{\prime}+k_{z^{\prime}} z^{\prime}\right)} \mathrm{d} x^{\prime} \mathrm{d} z^{\prime} .
\end{aligned}
$$

By performing an inverse Fourier transform of the recombination dipole moment, one can now express the molecular orbital as:

$$
\psi_{0}^{u}\left(x^{\prime}, z^{\prime}\right)=\frac{\mathcal{F}_{\mathbf{k} \rightarrow \mathbf{r}^{\prime}}\left[d_{u}^{\hat{\mathbf{r}}}\left(k_{x^{\prime}}, k_{z^{\prime}}\right)\right]}{u},
$$

while in the velocity form, where the dipole operator is $\hat{\mathbf{d}}^{\hat{\mathbf{p}}}=-\mathrm{i} \nabla_{\mathbf{r}^{\prime}}$, the orbital is expressed as:

$$
\psi_{0}^{u}\left(x^{\prime}, z^{\prime}\right)=\mathcal{F}_{\mathbf{k} \rightarrow \mathbf{r}^{\prime}}\left[\frac{d_{u}^{\hat{\mathbf{p}}}\left(k_{x^{\prime}}, k_{z^{\prime}}\right)}{k_{u}}\right] .
$$


Due to the different approximations and in particular the SFA, the two forms are not equivalent. While there is no consensus on which form gives more precise orbital reconstructions, there may be technical reasons to prefer one or the other. For instance, if $\psi_{0}$ has a nodal plane containing the X' or z' axis (like $\pi_{g}, \pi_{u}, \sigma_{u}$ orbitals), one will run into numerical problem when dividing by $x^{\prime}$ and $z^{\prime}$ in Eq. 5. This problem is absent in the velocity form, since one divides by $k_{u}$.

The characterization of the harmonic emission provides points in the Fourier space, located at the positions $\left(k_{x^{\prime}}, k_{z^{\prime}}\right)$ defined by i) the energy conservation law, Eq. 2, where $k^{2}=k_{x^{\prime}}^{2}+k_{z^{\prime}}^{2}$; ii) the re-collision angle $\theta$. For a given $\theta$, the harmonic spectrum gives access to a slice in one quadrant of the Fourier space. The inverse spatial Fourier transform of such a slice corresponds to a $2 \mathrm{D}$ projection of the molecular orbital onto the perpendicular plane with respect to the direction given by $\theta$; this is the Fourier slice theorem. By exploring the whole Fourier space, i.e. changing the re-collision angle $\theta$ from 0 to $2 \pi$, the orbital is probed from all directions, allowing the reconstruction of the $2 \mathrm{D}$ orbital in real space. For symmetric molecules, which is the case for $\mathrm{N}_{2}$ considered here, one needs to measure $\theta$ from 0 to $\pi / 2$, and to determine the orbital symmetry. Its measurement requires control over the trajectory of the tunnel ionized electron in HHG, to transfer the information on a possible sign change between the lobes of the orbital into the harmonics' phase or polarization. It is probable that an additional second order harmonic laser beam with controllable delay with respect to the driving laser and polarized perpendicular to it meets the requirements [26]. Another approach for the symmetry determination is based on the a priori knowledge of the relation between the orbital symmetry and the polarization map of the harmonics, as proposed by [25].

The 2D reconstructions performed using the dipoles along the $x^{\prime}$ and $z^{\prime}$ axes, $\psi_{0}^{x^{\prime}}\left(x^{\prime}, z^{\prime}\right)$ and $\psi_{0}^{z^{\prime}}\left(x^{\prime}, z^{\prime}\right)$, should in principle be equivalent. However, due to the discrete angular and frequency sampling of the Fourier space and to the approximations, this is in general not the case. Therefore, we choose to define the reconstructed molecular orbital as the average of the two components:

$$
\psi_{0}\left(x^{\prime}, z^{\prime}\right)=\frac{1}{2}\left(\psi_{0}^{x^{\prime}}\left(x^{\prime}, z^{\prime}\right)+\psi_{0}^{z^{\prime}}\left(x^{\prime}, z^{\prime}\right)\right) .
$$

The proposal of tomographic orbital reconstruction raised a lot of scientific discussions [63, 64, 65, 62] about its interpretation, generality and the errors caused by the approximations, and in particular, by the PWA.
Another effect that sparked much debate is the presence of multi-orbital contributions. Further on, we concentrate on this problem and investigate two experimental approaches to overcome this problem.

\subsection{Multi-Orbital Contributions to $H H G$}

In contrast to rare-gas atoms, in molecules, the separation of the highest occupied energy levels may be reduced to few eVs, like in $\mathrm{N}_{2}: \Delta I_{\mathrm{p}}=I_{\mathrm{p}}(H O M O)-$ $I_{\mathrm{p}}(H O M O-1) \approx 1.4 \mathrm{eV}$. When such a molecule is exposed to high laser intensities, tunnel ionization may occur from different orbitals simultaneously creating different ionization channels [39, 33]. The channel that connects the neutral ground state to the ionic ground state (the $\mathrm{X}$ state obtained by ionizing from the HOMO in Koopmans' approximation) is called X channel, while the channel that leads to the first excited ionic state (the A state obtained by ionizing from the HOMO1 ) is called A channel. Therefore, the tunnel ionized electron leaves its parent ion in a coherent superposition of these states. In a first approximation to describe HHG from this multi-channel system, we neglect the coupling, relaxation or excitation between the channels. Therefore, the molecular dipole reads in the length form:

$$
\mathbf{D}\left(\omega, I_{L}, \theta\right)=\sum_{i} \gamma_{i}\left(\omega, I_{L}, \theta\right) a_{i}\left(\omega, I_{L}\right) \mathbf{d}_{i}^{\hat{\mathbf{r}}}(\omega, \theta)
$$

where $i=\mathrm{X}, \mathrm{A}$. The weight of each channel is given by the product of the tunnel ionization $\left(\gamma_{i}\right)$ and the continuum acceleration $\left(a_{i}\right)$ amplitudes. The lower lying orbital has a higher ionization potential than the HOMO so that the Keldysh exponential factor in $\gamma_{A}$ is smaller than in $\gamma_{X}$. However, the electrons tunnel ionize more easily along the most spatially extended component of the orbital. Therefore, the difference in the geometry of the two orbitals will play an important role through $R(\theta)$. In the case of $\mathrm{N}_{2}$, the HOMO has a maximum probability to ionize when the molecule is aligned parallel to the driving laser field $\left(\theta=0^{\circ}\right)$ and a minimum in the perpendicular case $\left(\theta=90^{\circ}\right)$. The situation is opposite for HOMO-1 that has a nodal plane along the molecular axis resulting in minimum probability at $0^{\circ}$ but a large probability at $90^{\circ}$. It is around this angle that the HOMO-1 contribution is expected to become significant. More generally, for higher-order harmonics with frequencies beyond the cut-off for the HOMO, the contribution of the HOMO-1 should be favored, due to its larger ionization potential [39]. This should result in an increase of the relative contribution of the continuum amplitude $a_{A}$ with respect to $a_{X}$. 


\section{Experimental Methods}

The application of the principles of molecular orbital tomography in the experiments is very demanding. The difficulty comes from the fact that one has both to control finely the alignment of the molecules and to characterize thoroughly the harmonic emission. In our setup, this requires the fine control of three different laser beams in two Mach-Zender interferometers. All three beams are focused into the $\mathrm{N}_{2}$ supersonic gas jet with a $\mathrm{f}=1 \mathrm{~m}$ lens. The first beam induces non-adiabatic molecular alignment [56, 57, 58]. The $120 \mathrm{fs}$ laser pulse, propagating collinearly with the generating (driving) pulse $\left(\mathrm{y}=\mathrm{y}^{\prime}\right)$, and focused at $\mathrm{I} \approx 0.5 \times 10^{14} \mathrm{~W} / \mathrm{cm}^{2}$, creates a rotational wave-packet which rephases periodically, aligning the molecules along its polarization (z') that makes an angle $\theta$ with the generating beam polarization (z).

The second beam, which is annular and of 50-fs duration, is focused at an intensity that can be varied in the range $0.7-1.3 \times 10^{14} \mathrm{~W} / \mathrm{cm}^{2}$ with a combination of a polarizer and a half-wave plate. It induces $\mathrm{HHG}$ at the half-revival of the rotational wave-packet. The detected harmonic signal is then the result of a coherent averaging of the single molecular response over the alignment distribution of the molecules at half revival.

A precise determination of the angular dependence of the single molecule radiating dipole requires in principle to deconvolve the macroscopic signal from this alignment distribution, except in the case of a high degree of alignment $[59,60]$. In our experimental conditions, we estimate the degree of alignment: $\left\langle\cos ^{2} \psi\right\rangle \approx$ 0.6 , where $\psi$ is the molecular angle with respect to the aligning laser polarization. This corresponds approximately to a width of the alignment distribution of about $30^{\circ}$. In the following, we do not deconvolve the experimental data from this distribution.

The third beam, called dressing beam, is focused at a moderate intensity $\left(\approx 10^{11} \mathrm{~W} / \mathrm{cm}^{2}\right)$ that does not perturb the harmonic emission. It propagates along with the driving and aligning beams and while the latter two are cut out by an iris, it is refocused together with the harmonic beam into the detection chamber where they induce 2-photon XUV+IR ionization of a target gas. The photoelectrons are detected with a magnetic bottle electron spectrometer. In this article, we perform the measurement of the spectral amplitude and phase of the $\mathrm{z}$ component of the dipole (i.e., the component parallel to the generating laser), that was preferentially reflected (s polarization) by two gold-coated mirrors. Characterizing also the -small- $\mathrm{x}$ (orthogonal) component is extremely difficult since it would require combining the above techniques with polarimetry at the expense of the number of harmonic photons [61].

The phase characterization is performed using the Reconstruction of Attosecond Beating By Interference of two-photon Transition (RABBIT) technique [8]. It yields the group delay (GD) at the even ordered sidebands (SB): $\mathrm{GD}_{q}=\left(\phi_{q+1}-\phi_{q-1}\right) / 2 \omega_{0}$, where $\phi_{q \pm 1}$ are the phases of two consecutive odd harmonics and $\omega_{0}$ is the fundamental laser frequency. RABBIT detects only the spectral phase but it is not able to measure the phase variation with alignment angle at a fixed harmonic order nor the absolute phase difference with Ar. Our calibration procedure imposes that (1) the phase of harmonic 15 is the same as in Ar for all $\mathrm{N}_{2}$ alignments and for all intensities. By doing so, we add an $\omega$-independent phase term $\varphi_{0}(\theta)$. Hence it does not change the spectral characteristics of the dipole. Next, it imposes (2) the same phase value for harmonic 17 (which is a consequence of (1) plus the GD normalization at side band 16). This is the consequence of the instability in absolute timing measurements: all the GD curves are then normalized at sideband 16 to a typical GD value of Ar. (2) results in the removal of a linear phase as a function of harmonic order that is specific to each laser intensity and alignment angle. This is justified by the measurements of only small variations of the low order harmonic phase with $\theta$ [79] using a two-source interferometry technique $[33,13]$. Therefore, the variations of the total dipole phase are modified by a small linear term, but they are not washed out. Finally, we used the GDs measured in Ar to determine the effective generating laser intensity in the gas jet, since the slope (GDD $\Delta t_{\mathrm{e}}$ related to the attochirp) of the plateau region is directly connected to $\mathrm{I}_{L}[78]$.

The orbital tomography is derived in the molecular frame; therefore, our measured RDM has to be transformed to it from the laboratory frame. By assuming that the induced dipole has a negligible $\mathrm{x}$-component, the RDM writes in the molecular frame ( $\left.x^{\prime}, y^{\prime}, z^{\prime}\right)$ as follows:

$$
\begin{aligned}
& d_{x^{\prime}}^{f}=d_{\|}^{f} \sin \theta \\
& d_{z^{\prime}}^{f}=d_{\|}^{f} \cos \theta,
\end{aligned}
$$

where $d_{z^{\prime}}^{f}$ is induced along the main molecular axis while $d_{x^{\prime}}^{f}$ is perpendicular to it. 


\section{Results and discussion}

\subsection{Separation of the Contributions from the HOMO and $\mathrm{HOMO}-1$ in $\mathrm{N}_{2}$}

The questions that now arise are: (a) How do the two orbital contributions interfere in the total harmonic signal? and more interestingly (b) how do they manifest in the tomographic reconstruction? Is it still possible to reconstruct a meaningful orbital? In our procedure of dipole extraction (see Eq. 3), we calibrated the measured $\mathrm{N}_{2}$ harmonic signal with the measured $\mathrm{Ar}$ signal to remove the tunnel ionization and the continuum acceleration factors. However, this calibration is not valid for the HOMO-1 since its ionization potential is not equal to the one of Ar. Therefore, the calibration process does not remove the amplitude ratio and phase difference terms between the HOMO and the HOMO-1.

The dipole phase is the most crucial element for the tomographic reconstruction, as dictated by the importance of the phase for the outcome of a Fourier transform. So let us study the phase difference between the HOMO-1 and the HOMO contributions in Eq. 8:

$$
\Delta \varphi_{\text {tot }}(\omega)=\Delta \varphi_{\text {ion }}(\omega)+\Delta \varphi_{\text {con }}(\omega)+\Delta \varphi_{\text {rec }}(\omega)
$$

The most common tunnel ionization models [50, 53, 66] do not contain phase terms. Note that recent studies, like [67] and [68], are discussing the possibility of a tunnel ionization phase, though mostly in the context of coupled ionization channels. Therefore, we assume that $\Delta \varphi_{\text {ion }}(\omega)=0$.

During the second stage of the process, the electrons in the continuum are mainly governed by the driving laser. Using the saddle-point approximation for the molecular dipole, it can be shown that [34, 43, 69]: $\Delta \varphi_{\text {con }}(\omega) \approx-\Delta I_{\mathrm{p}} \tau_{\omega}$, where $\tau_{\omega}$ is the average excursion time of the trajectory corresponding to the emission of harmonic $\omega . \Delta \varphi_{\text {con }}(\omega)$ can be controlled through the intensity of the driving laser $\left(I_{L}\right)$, since it affects the excursion time of the EWP. As shown in Haessler et al. [34], this term varies over the observed spectral range at a given $I_{L}$ and the phase difference for the 25th harmonic order at $I_{L}=1.2 \times 10^{14} \mathrm{~W} / \mathrm{cm}^{2}$ is $\Delta \varphi_{\text {con }}\left(25 \omega_{0}\right) \approx-\pi$, varying slowly by $\pm 0.2 \pi$ between harmonics 17 and 31 .

In the PWA, the symmetry of the molecular orbitals, the dipole form and the properties of the Fourier transform directly determine the RDM. The HOMO and HOMO- 1 orbitals of $\mathrm{N}_{2}$ have $\sigma_{g}$ and $\pi_{u}$ symmetry, respectively. In the length form, the HOMO PW dipole is then purely imaginary valued, $\varphi_{\text {rec }}^{\mathrm{HO}}(\omega)=\pi / 2$, while the HOMO-1 PW dipole is purely real valued, $\varphi_{\text {rec }}^{\mathrm{HO}-1}(\omega)=\pi$. Hence, the two are shifted in phase by $\Delta \varphi_{\text {rec }}(\omega)=\pi / 2$. In [34], it was shown that this conclusion still holds for Coulomb Wave (CW) dipoles in the limited experimentally-accessible spectral range. When considering Scattering Wave (SW) dipoles, this can be considered a crude but still meaningful approximation in the same limited spectral range.

Therefore, in these specific generation conditions, $\Delta \varphi_{\text {tot }} \approx-\frac{\pi}{2}$ in the considered spectral range. The answer to question (a) is thus that the two orbital contributions are approximately disentangled in the real and imaginary parts of the measured dipole. The answer to question (b) is that the HOMO orbital can be reconstructed from the imaginary part, while the HOMO-1 from the real part of the measured recombination dipole moment (provided that an additional rotation in the complex plane is performed to account for the Coulomb phase shift).

Figures 1(e-f) show the experimental reconstructions for the HOMO and HOMO-1 orbitals performed at $I_{L}$ $=1.2 \times 10^{14} \mathrm{~W} / \mathrm{cm}^{2}$ (from [34]). The emission from $\mathrm{N}_{2}$ molecules rotated from $0^{\circ}$ to $90^{\circ}$ with a step size of $10^{\circ}$ was characterized in the spectral range covering harmonic 17 to 31 . For comparison, we plot in Fig. 1(a-b) the Hartree-Fock (HF) HOMO and HOMO1 orbitals, and in (c-d), these orbitals "filtered" in the experimental spectral range. More precisely, the PW dipole for each orbital was filtered to account for the limited harmonic range H17-31, that corresponds to 1.39a.u. $<k<1.88$ a.u. using the heuristic relation: $\omega=\frac{k^{2}}{2}$. The latter is used instead of Eq. 2 in order to take into account the additional acceleration of the returning EWP in the ion field that is missing in the PWA. The tomographic procedure was then applied to the filtered dipoles to recover the orbitals. For both the HOMO and HOMO-1 experimental reconstructions, the main distortions compared to the HF orbitals are coming from the limited experimental spectral range, as shown by the good agreement with the filtered HF orbitals. In particular for the HOMO, the three lobes with alternating signs are well recovered in the experimental reconstruction. This demonstrates the feasibility of accessing both the amplitude and the phase of valence orbitals.

The above reconstructions have been made possible by the fact that: $\Delta \varphi_{t o t} \approx-\frac{\pi}{2}$. When changing the generation conditions, and in particular $I_{L}, \Delta \varphi_{\text {tot }}$ deviates from $-\pi / 2$ and mixes the two contributions. In order to illustrate this point, we present in Figs $1(\mathrm{~g}-\mathrm{h})$ reconstructions performed at $\mathrm{I}_{L}=1.0 \times 10^{14} \mathrm{~W} / \mathrm{cm}^{2}$. While the 


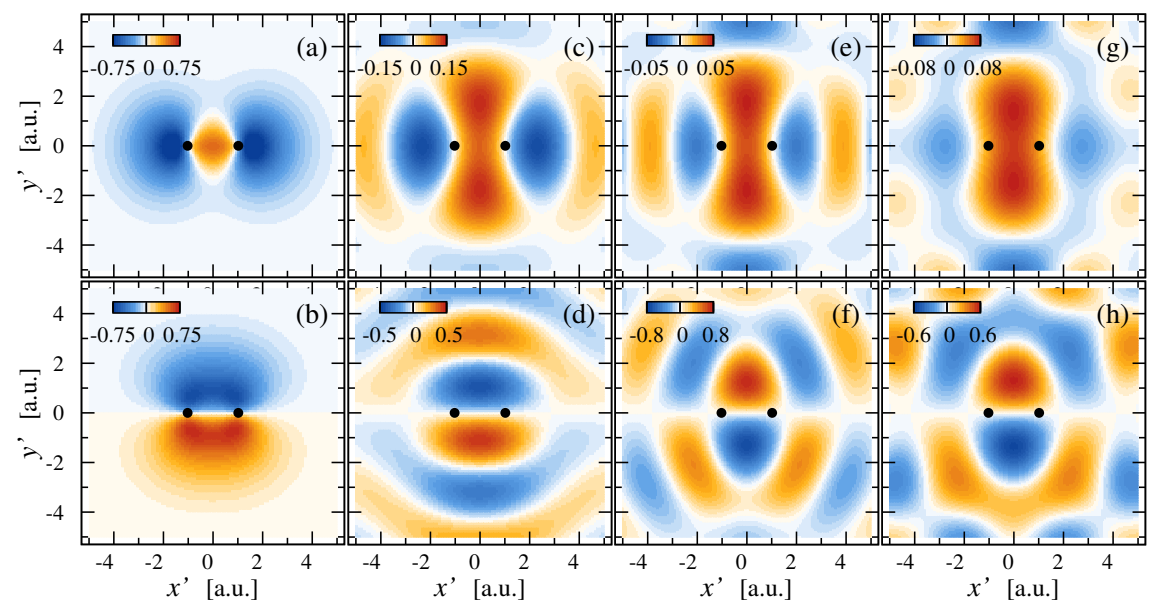

Figure 1: Orbital tomographic reconstructions. Hartree-Fock calculations for the HOMO (a) and HOMO-1 (b) of $\mathrm{N}_{2}$ molecules. The same theoretical simulations filtered for the experimental spectral range H17-31 for HOMO (c) and HOMO-1 (d). HOMO (e) and HOMO-1 (f) orbitals reconstructed from the experimentally characterized dipole in the H17-31 spectral range at $\mathrm{I}_{L}=1.2 \times 10^{14} \mathrm{~W} / \mathrm{cm}^{2}$ (from [34]). HOMO (g) and HOMO-1 (h) orbitals reconstructed at $\mathrm{I}_{L}=1.0 \times 10^{14} \mathrm{~W} / \mathrm{cm}^{2}$. Each figure is normalized separately.

HOMO central lobe is weakly affected, the two side lobes have a much less negative amplitude and additional lobes appear in the direction perpendicular to the molecule. This demonstrates how sensitive the reconstruction is to the generation conditions. One may even reach $\Delta \varphi_{t o t} \approx-\pi$ leading to a destructive interference, so-called dynamical interference, already observed in $\mathrm{CO}_{2}$ [33]. Furthermore, in the general case, one may have more than two orbitals contributing to the harmonic signal, again like in $\mathrm{CO}_{2}$ where HOMO-2 also contributes, preventing the use of the above "trick" for performing tomography. It is thus crucial to find conditions where a single orbital is contributing. Intuitively, one may think that by decreasing the intensity of the driving laser, the probability of tunnel ionization from the lower orbitals should decrease faster than for the HOMO which may at some point become dominant. However, before applying the tomographic procedure, we need a criterion to decide whether we have single or multi-orbital (channel) contributions.

\subsection{Selection of the HOMO contribution in $\mathrm{N}_{2}$}

\subsubsection{Study of the minimum in the HHG spectra}

We may first consider the calibrated harmonic intensity for different values of $I_{L}$ and look for a dynamical interference: the destructive interference should result in a minimum in the harmonic spectrum [33]. Changing $I_{L}$ modifies $\Delta \varphi_{\text {tot }}$ and results in a shift of the spectral position of the minimum. Such an $I_{L}$-dependent minimum position is a signature of multi-orbital HHG. Fig. $2 \mathrm{a}$ and $\mathrm{b}$ show the calibrated intensity of the total dipole measured in $\mathrm{N}_{2}$. The molecules were aligned (at the half-revival of the rotational wave-packet) parallel $\left(\theta=0^{\circ}\right)$ and perpendicular $\left(\theta=90^{\circ}\right)$ to the driving laser polarization. Due to the symmetry properties of the two orbitals, two extreme cases can be studied: when mainly the HOMO is contributing (parallel case) and when both HOMO and HOMO-1 are present (perpendicular case). For both alignments, at all intensities, there is a clear minimum in the spectra, almost at the same harmonic order $\mathrm{H} 23(36 \mathrm{eV})$. While the minimum does not shift when increasing $I_{L}$, it gets slightly deeper. Relying only on these results, one would conclude that this minimum is related to a destructive interference occurring in a single HHG channel (Cooper-like minimum). But we cannot exclude the possibility of multiple orbital contributions not sufficiently dephased to produce a clear interference in the investigated $I_{L}$ range.

These results are consistent with those presented in the literature. Several groups have observed this minimum for different driving laser intensities, wavelengths and molecular alignments $[70,71,72,73]$, but the minimum was not observed to move significantly, even though the exact position differed slightly within a few eV range. Farrell et al. [74] could observe a slightly moving minimum in the spectrum between $\mathrm{H} 23$ and $\mathrm{H} 27$ by changing both the intensity and the molecular alignment. While relating it to multi-orbital contributions, they recognized that a "field-free static model of the molecule is insufficient to explain the observations". Mairesse et al. [73] attributed it tentatively to multi-electron effects. Two theoretical studies reported 


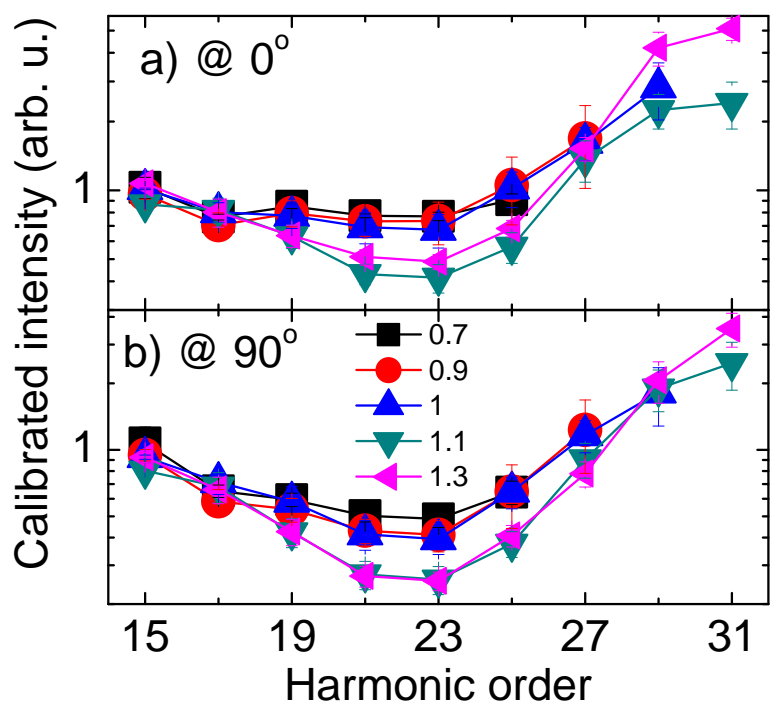

Figure 2: Calibrated harmonic intensities for $\mathrm{N}_{2}$ molecules aligned parallel (a) and perpendicular (b) to the driving laser. The laser intensities are expressed in units of $10^{14} \mathrm{~W} / \mathrm{cm}^{2}$. The error bars show the standard deviation of about five measurements; their size is close to the size of the data symbols.

a spectral minimum. First, a minimum at $45 \mathrm{eV}$ was reported by Jin et al. [75] in an effort to explain the results shown in Wörner et al. [72], using only the HOMO and including macroscopic effects. The minimum resulted from a change of sign of the scattering-wave HOMO $\mathrm{RDM}$ at $50 \mathrm{eV}$ and $\theta=0^{\circ}$ and the angular averaging shifted the minimum towards lower energy. More recently, they showed that a minimum (around $38 \mathrm{eV}$ ) could also be obtained at $\theta=90^{\circ}$ due to the fast dropoff of the HOMO RDM at this angle [82]. The combination with the macroscopic wavepacket for a 1200-nm driving laser resulted in a slight shift of the minimum with increasing $I_{L}$. At high $I_{L}$, the minimum disappears due to the emergence of the HOMO-1 contribution. Another interpretation for the spectral minimum was proposed by O. Smirnova [76, 77]: the spectral minimum would originate from the temporal filtering of the recombination dipole moment due to the attosecond timescale of the emission process. The shape resonance occurring in $\mathrm{N}_{2}$ around $30 \mathrm{eV}$ could result in a spectral minimum at a higher energy when filtered on an attosecond time scale. This would mean that the recombination dipole recovered from HHG would not simply be the complex conjugate of the photoionization dipole. Up to now, there is no consensus in the community on the origin of the $\mathrm{N}_{2}$ minimum.

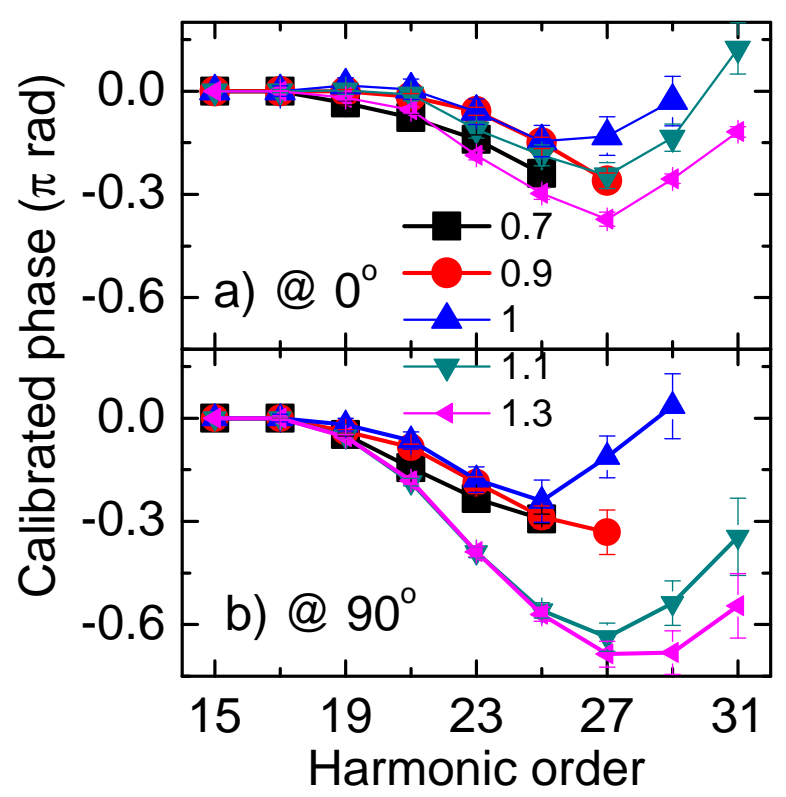

Figure 4: Calibrated harmonic phases for $\mathrm{N}_{2}$ molecules aligned parallel (a) and perpendicular (b) to the driving laser. The color codes and corresponding intensities are the same as in Fig. 3. The plotted phases are an average over 5 calibrated phase curves measured with the same $\mathrm{I}_{L}$. The error bars are calculated from the calibration procedure; their size at low order is comparable or smaller than the data symbols. The phases at $\mathrm{H} 15$ and $\mathrm{H} 17$ are set to 0 by the calibration procedure (see text).

\subsubsection{Study of the calibrated HHG spectral phase}

This shows the need for more sources of information. In the following, we analyze the spectral phase of the harmonic emission instead of the spectral amplitude. We show that the calibrated spectral phase is more sensitive to changes in the intensity of the driving field.

Figures $3 \mathrm{a}, \mathrm{b}, \mathrm{c}$ show the measured group delays for different $\mathrm{I}_{L}$ in Ar and $\mathrm{N}_{2}$ aligned parallel and perpendicular to the driving laser field, respectively. These are typical data for each laser intensity value, collected on different days. It was not feasible to acquire all data in one measurement run, because we have chosen a more precise, but rather slow scanning strategy: instead of making relatively fast series of RABBIT scans with varying intensity for each gas and alignment angle, we chose to perform, for any given intensity, the three RABBIT measurements in $\mathrm{Ar}$ and $\mathrm{N}_{2}\left(\theta=0^{\circ}, 90^{\circ}\right)$ in a row. This assures that the calibration by the reference atom is correct (i.e. done with an Ar measurement under the precisely equal experimental conditions). While this strategy gives more precise results, it is much slower to implement (a triplet of RABBIT scans for one intensity takes about 1 hour). The calibration by the reference atom should, however, remove any influences com- 


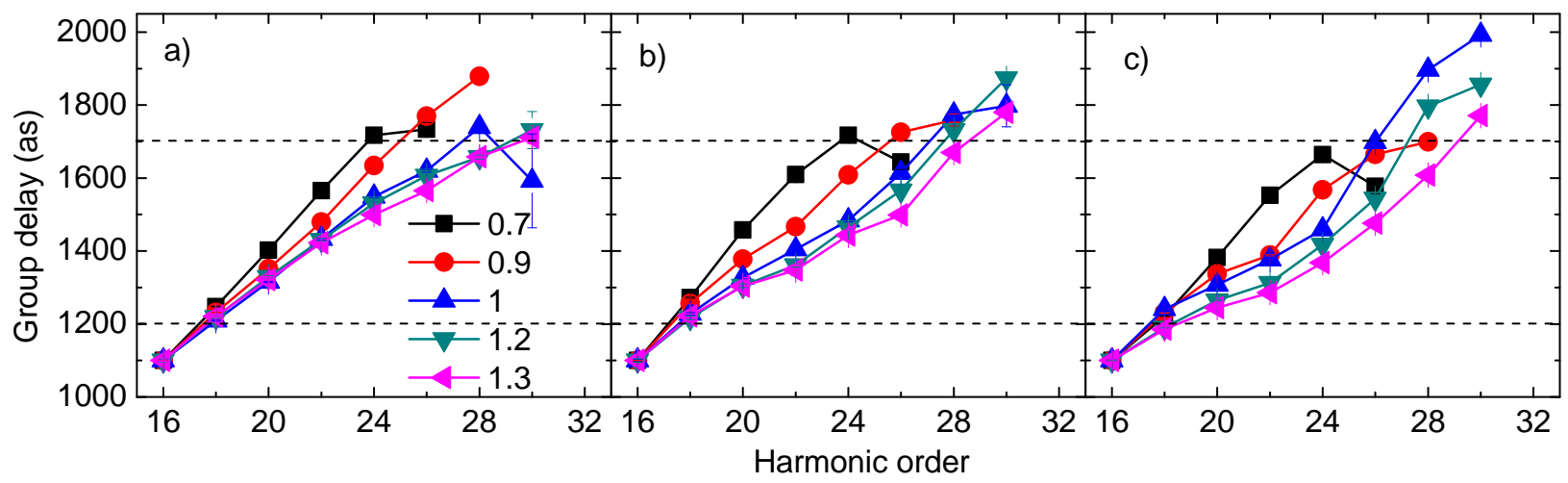

Figure 3: Measured group delays of $\operatorname{Ar}(\mathrm{a}), \mathrm{N}_{2}\left(\theta=0^{\circ}\right)(\mathrm{b})$ and $\mathrm{N}_{2}\left(\theta=90^{\circ}\right)$ (c). The code of the symbols and the corresponding $\mathrm{I}_{L}$ values (in units of $10^{14} \mathrm{~W} / \mathrm{cm}^{2}$ ) are given in (a). Horizontal dashed lines are drawn at 1200 as and 1700 as to make easier the comparison between the plots. The error bars are retrieved from the numerical analysis of the RABBIT trace; their size is comparable to the size of the data symbols.

ing from the setup alignment, focusing geometry, laser beam profile, etc., which may drift from day to day.

Three main observations may be drawn from Fig. 3: (i) At low intensity $\left(I_{L}=0.7 \times 10^{14} \mathrm{~W} / \mathrm{cm}^{2}\right)$, the three GD curves in $(a, b, c)$ are very similar to each other. This appears clearly when they are plotted on top of each other, as shown in Fig. 5a). (ii) At higher intensity, for $\mathrm{N}_{2}$ (b,c), a dip appears for orders in the plateau region around harmonics 23-25, which increases with $I_{L}$ and is always larger for perpendicular than for parallel alignment. (iii) At high $I_{L}$, towards the cut-off energies around side bands 26-30 in (b,c), the GD steeply increases for $\theta=0^{\circ}$ and even more for $\theta=90^{\circ}$. Such an increase is absent in the case of Ar, which rather shows the sign of saturation related to the cut-off harmonics. All the effects discussed in (i-iii) are transferred to the phase of $\mathrm{N}_{2}$ calibrated by the one of Ar, Fig. $4 \mathrm{a}, \mathrm{b}$. At the lowest intensities, the phase seems to be similar for both alignments (i). At higher intensity, the GD dip (ii) corresponds to a phase decrease around harmonics 2327 , followed by a phase increase (iii) at high orders 27 31. At $\theta=0^{\circ}$ (fig $4 \mathrm{a}$ ), there is little variation with $I_{L}$ : all curves are grouped together. In contrast, at $\theta=90^{\circ}$ (fig $4 \mathrm{~b}$ ), there is a strong variation when $I_{L}$ reaches $1.1 \times 10^{14} \mathrm{~W} / \mathrm{cm}^{2}$.

\subsubsection{Discussion}

In order to understand the physical origin of the phenomena observed in Fig. 3 and 4, we have derived theoretically the characteristics of the observed GDs in the case where a single orbital is contributing. Each of the three steps of HHG contributes to the total phase and we wrote from Eq. 11: $\varphi_{\text {tot }}(\omega)=\varphi_{\text {ion }}(\omega)+\varphi_{\text {con }}(\omega)+$ $\varphi_{\text {rec }}(\omega)$. Assuming that the ionization phase is zero (see above), the group delay is obtained by deriving the total phase with respect to the frequency:

$$
G D(\omega)=\frac{\partial \varphi_{t o t}(\omega)}{\partial \omega} \approx \frac{\partial \varphi_{c o n}(\omega)}{\partial \omega}+\frac{\partial \varphi_{r e c}(\omega)}{\partial \omega}
$$

This means that the GD is determined by two terms: $\mathrm{GD}_{\text {con }}$, the delay in the recombination time of the electron trajectory corresponding to each harmonic order, related to the different excursion times, and $\mathrm{GD}_{\text {rec }}$, that is associated to the phase of the RDM. The first term is intensity-dependent and species-independent (for simi$\operatorname{lar} I_{p} \mathrm{~s}$, within SFA); the second is intensity-independent and species- and $\theta$ - dependent. Fig. 5 presents both terms for $\mathrm{Ar}$ and the $\mathrm{N}_{2} \mathrm{HOMO}\left(\theta=0^{\circ}, 90^{\circ}\right)$. The HOMO RDM were calculated using exact scattering waves [23] and averaged over the experimental alignment distribution. In the plateau region, $\mathrm{GD}_{\text {con }}$ increases linearly with harmonic order $\approx \Delta t_{\mathrm{e}} \omega$ for short trajectories and saturates in the cutoff region. The $\mathrm{GD}_{\text {rec }}$ are slightly different for $\mathrm{Ar}, \mathrm{N}_{2}$ at $0^{\circ}$ and $90^{\circ}$, with absolute values around 0 as, 80 as and 40 as respectively. They do not vary much over the considered spectral range (partly due to the angular averaging), so that they mainly result in a global shift of the total GD. However, this shift is lost when we normalize the total GD curves to the same value (1100 as) at SB16, and all three curves just fall on top of each other as shown in Fig. $6 \mathrm{~b}$ at an intensity of $\mathrm{I}_{L}=0.7 \times 10^{14} \mathrm{~W} / \mathrm{cm}^{2}$. This is true for all intensity values since $\mathrm{I}_{L}$ only changes the species-independent $\mathrm{GD}_{\text {con }}$, as long as a single orbital (here the HOMO) contributes to the emission. Any deviation between the three curves when varying $I_{L}$ can thus be directly attributed to the appearance of a significant HOMO-1 contribution.

The comparison with the experimental results obtained at $\mathrm{I}_{L}=0.7 \times 10^{14} \mathrm{~W} / \mathrm{cm}^{2}$ (Fig. $6 \mathrm{a}$, corresponding 


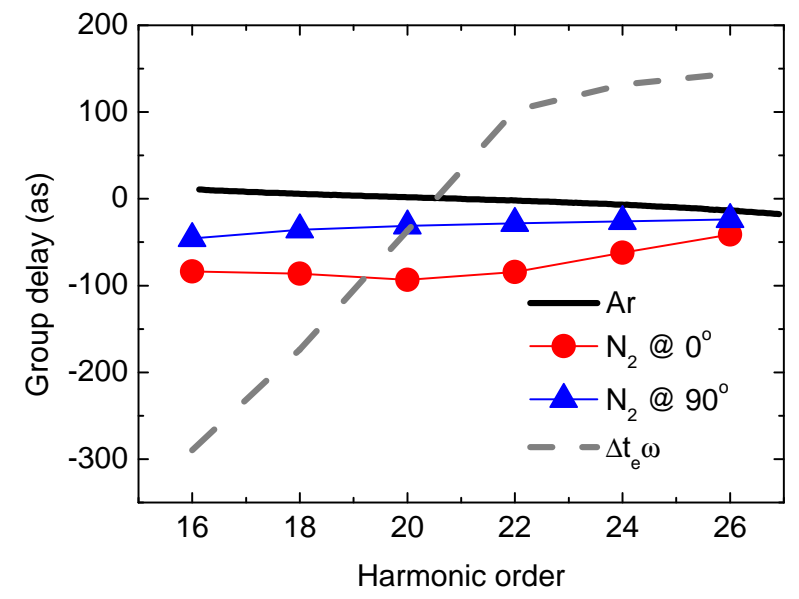

Figure 5: Derivative of the RDM phases with respect to the harmonic frequency $\partial \varphi_{\text {rec }}(\omega) / \partial \omega$ for Ar (black line), $\mathrm{N}_{2} \mathrm{HOMO}$ at $\theta=0^{\circ}$ (red squares) and $\theta=90^{\circ}$ (blue triangles). The gray dashed line represents the continuum group delay $\partial \varphi_{c o n}(\omega) / \partial \omega$ calculated with SFA at $\mathrm{I}_{L}$ $=0.7 \times 10^{14} \mathrm{~W} / \mathrm{cm}^{2}$; it is shifted by -1700 as for better visibility. The scattering-wave RDM of $\mathrm{N}_{2}$ are averaged over the alignment distribution. The GD for Ar is retrieved from Jin et al. [75].

to point (i)) demonstrates that there is very little contribution from the HOMO-1 in this low intensity regime. It is mainly $\mathrm{GD}_{\text {con }}$ that determines the behavior of the GD curve while the RDM does not add any substantial GD. The non-linear behavior of tunnel ionization at low intensity thus efficiently selects the HOMO orbital contribution.

Note that there is a difference at high orders between measurements and calculations in Fig. 6: the measured group delays reach the cut-off saturation one order higher than the theoretical ones. One possible explanation relies in the SAE and single molecule calculations. Propagation effects are not taken into account which may contribute to the total dipole phase.

As a consequence of the above discussion, plotting the calibrated phase allows to remove $\varphi_{c o n}$ and thus any $\mathrm{I}_{L}$-dependent term as long as a single orbital is contributing. This is approximately the case at $\theta=0^{\circ}$ in Fig. $4 \mathrm{a}$ since there is little evolution of the calibrated phase when varying $\mathrm{I}_{L}$. This is also true at $\theta=90^{\circ}$ for low intensity. However, for $\mathrm{I}_{L}>0.9 \times 10^{14} \mathrm{~W} / \mathrm{cm}^{2}$, the strong deviation of the calibrated phase in Fig. $4 \mathrm{~b}$ (point (ii)) indicates the presence of multi-orbital contributions, the interference of which is sensitive to the laser intensity.

The emergence of the HOMO-1 contribution alone cannot explain the re-increase of the phase for higher harmonics observed in Fig. 4, point (iii). This could be interpreted as resulting from the nuclear dynamics, as

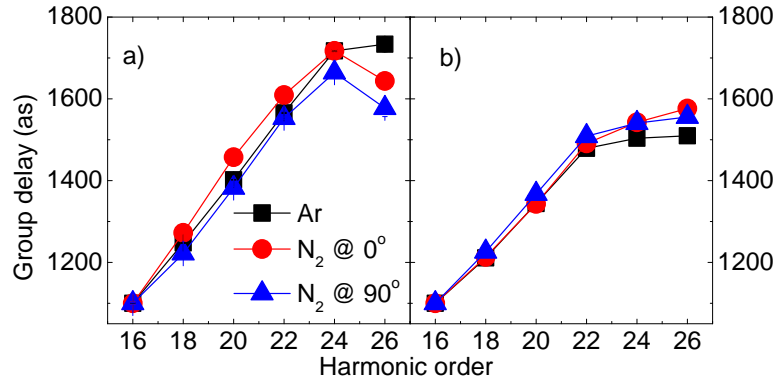

Figure 6: Total group delay for $\mathrm{I}_{L}=0.7 \times 10^{14} \mathrm{~W} / \mathrm{cm}^{2}$ of $\mathrm{Ar}$ (black square), $\mathrm{N}_{2}$ parallel (red circle) and perpendicular (blue triangle) alignment measured experimentally (a) and calculated (b) from the values presented in figure 5. All curves are normalized to 1100 as at sideband 16 .

shown by the modeling of our experiments [41]. The equilibrium distance of the ion in the A state is significantly different from that of the neutral molecule (and of the $\mathrm{X}$ state), so that ionization through this channel results in the coherent population of many vibrational states that induces ultrafast nuclear dynamics on the sub-fs time scale $[28,29,30]$. Consequently, the fast evolution of the nuclear autocorrelation function in channel A both decreases its contribution to the HHG and introduces an additional phase term, resulting in the re-increase of the phase (therefore of the GD) at high harmonic orders. Indeed, these orders correspond to the longest excursion times in the continuum (when the short trajectories are selected like in our experiment). This opens up the possibility to study the ultrafast nuclear dynamics in molecules, for instance by changing the wavelength of the driving laser while keeping all the other parameters constant. This would allow to control the excursion time of the continuum EWP, and therefore, to probe the evolved nuclear wave-packet at different recollision times resulting in a monitoring of the dynamics of the nuclear oscillation.

Before closing, we would like to stress the striking difference between the fast variation with $I_{L}$ of the calibrated spectral phase in Fig. $4 \mathrm{~b}$, and the weak $I_{L^{-}}$ variation of the corresponding calibrated intensity in Fig. $2 \mathrm{~b}$. The calibrated phase clearly shows that the phase jump over the spectrum is much smaller than $\pi$, which rules out destructive interference as a possible cause of the spectral minimum. The two effects (amplitude minimum and phase change) thus do not seem to be correlated and may have different origins. In any case, when the phase difference between two orbital contributions is of the order of $\pi / 2$, as it is the case here, phase measurements are a much more sensitive probe than intensity measurements. 


\section{Summary and conclusions}

We presented the principles of molecular orbital tomography and summarized the main difficulties that have to be overcome in order to extend it to other molecules or to pump-probe measurements.

We investigated the interesting phenomenon of multiple orbital contributions to the $\mathrm{HHG}$ in $\mathrm{N}_{2}$. Studying the harmonic spectral phase as a function of the driving laser intensity and molecular alignment, we showed that it is a more sensitive probe of multi-orbital effects than is the harmonic intensity. By exploiting the information encoded in the spectral phase, we found specific conditions in $\mathrm{N}_{2}$ where the HOMO and HOMO-1 contributions are disentangled in the imaginary and real parts of the dipole, respectively. This allowed us to perform a tomographic reconstruction of both orbitals. Finally, supported by experimental results, we introduced a general approach to measure and evidence a single orbital contribution to the HHG. The decrease of the driving laser intensity provides proper conditions for drastically reducing lower-lying orbital contributions through the selection by tunnel ionization. Unfortunately, it also results in both a lower signal-to-noise ratio and a lower cut-off for the generated harmonics, which decreases the spatial resolution of the tomographic reconstruction. We thus could not perform a tomographic reconstruction at $I_{L}=0.7 \times 10^{14} \mathrm{~W} / \mathrm{cm}^{2}$.

However, the use of driving lasers with longer central wavelength $\left(\lambda_{L}\right)$, like mid-IR (MIR) lasers, would overcome this problem, since the cut-off frequency of the generated harmonics scales as: $\omega_{\max } \sim I_{L} \lambda_{L}^{2}$. While keeping $I_{L}$ low enough not to induce multi-channel HHG, MIR lasers further increase the spatial resolution [60]. The main drawback could be the reduced dipole which scales as $\lambda_{L}^{-5}$ [80] and could prevent the measurement of the phase due to the low signal. However, phase retrieval algorithms [60] and improved phase matching conditions [81] may make it possible to perform tomographic reconstructions with high spatial resolution.

Even though the precision of the reconstructed orbitals is limited by the applied approximations like the PWA, we believe that tomographic reconstruction has an outstanding potential for measuring the temporal evolution of an orbital during, for instance, a chemical reaction. Observing the ultrafast distortions on the attosecond/femtosecond time scale would be a big step forward, even with a limited spatial resolution. Alternatively, chemical imaging with iterative techniques has been proposed theoretically to investigate, e.g., isomerization dynamics [64].

\section{Acknowledgements}

We thank R. Lucchese for providing us with the $\mathrm{N}_{2}$ RDM and P. Breger for his help in the experiments. We acknowledge financial support from the EU-FP7ATTOFEL and ANR-09-BLAN-0031-01.

\section{References}

[1] A. H. Zewail, Femtochemistry: Atomic-scale dynamics of the chemical bond, J. Phys. Chem. A 104 (24) (2000) 5660-5694.

[2] F. Krausz, M. Y. Ivanov, Attosecond physics, Rev. Mod. Phys. 81 (2009) 163.

[3] A. Mcpherson, G. Gibson, H. Jara, U. Johann, T. S. Luk, I. A. McIntyre, K. Boyer, C. K. Rhodes, Studies of multiphoton production of vacuum ultraviolet-radiation in the rare-gases, J. Opt. Soc. Am. B 4 (4) (1987) 595-601.

[4] M. Ferray, A. L'Huillier, X. Li, L. Lompre, G. Mainfray, C. Manus, Multiple-harmonic conversion of $1064 \mathrm{~nm}$ radiation in rare gases, J. Phys. B 21 (3) (1988) L31-L35.

[5] P. B. Corkum, Plasma perspective on strong field multiphoton ionization, Phys. Rev. Lett. 71 (13) (1993) 1994-1997.

[6] K. J. Schafer, B. Yang, L. F. Dimauro, K. C. Kulander, Above threshold ionization beyond the high harmonic cutoff, Phys. Rev. Lett. 70 (11) (1993) 1599-1602.

[7] M. Lewenstein, P. Balcou, M. Y. Ivanov, A. L'Huillier, P. B. Corkum, Theory of high-harmonic generation by low-frequency laser fields, Phys. Rev. A 49 (3) (1994) 2117-2132.

[8] P. M. Paul, E. S. Toma, P. Breger, G. Mullot, F. Auge, P. Balcou, H. G. Muller, P. Agostini, Observation of a train of attosecond pulses from high harmonic generation, Science 292 (5522) (2001) 1689-1692.

[9] M. Hentschel, R. Kienberger, C. Spielmann, G. Reider, N. Milosevic, T. Brabec, P. Corkum, U. Heinzmann, M. Drescher, F. Krausz, Attosecond metrology, Nature 414 (6863) (2001) 509-513.

[10] Y. Mairesse, F. Quéré, Frequency-resolved optical gating for complete reconstruction of attosecond bursts, Phys. Rev. A 71 (1) (2005) 011401.

[11] G. Sansone, E. Benedetti, F. Calegari, C. Vozzi, L. Avaldi, R. Flammini, L. Poletto, P. Villoresi, C. Altucci, R. Velotta, S. Stagira, S. De Silvestri, M. Nisoli, Isolated single-cycle attosecond pulses, Science 314 (5798) (2006) 443-446.

[12] M. Drescher, M. Hentschel, R. Kienberger, M. Uiberacker, V. Yakovlev, A. Scrinzi, T. Westerwalbesloh, U. Kleineberg, U. Heinzmann, F. Krausz, Time-resolved atomic inner-shell spectroscopy, Nature 419 (6909) (2002) 803-807.

[13] X. Zhou, R. Lock, W. Li, N. Wagner, M.M. Murnane, H.C. Kapteyn, Molecular Recollision Interferometry in High Harmonic Generation, Phys. Rev. Lett. 100 (2008) 073902.

[14] P. Tzallas, D. Charalambidis, N. A. Papadogiannis, K. Witte, G. D. Tsakiris, Direct observation of attosecond light bunching, Nature 426 (6964) (2003) 267-271.

[15] G. Sansone, F. Kelkensberg, J. F. Perez-Torres, F. Morales, M. F. Kling, W. Siu, O. Ghafur, P. Johnsson, M. Swoboda, E. Benedetti, F. Ferrari, F. Lepine, J. L. Sanz-Vicario, S. Zherebtsov, I. Znakovskaya, A. L'Huillier, M. Y. Ivanov, M. Nisoli, F. Martin, M. J. J. Vrakking, Electron localization following attosecond molecular photoionization, Nature 465 (7299) (2010) 763-766.

[16] S. Haessler, B. Fabre, J. Higuet, J. Caillat, T. Ruchon, P. Breger, B. Carré, E. Constant, A. Maquet, E. Mével, P. Salières, R. Taïeb, Y. Mairesse, Phase-resolved attosecond near-threshold 
photoionization of molecular nitrogen, Phys. Rev. A 80 (1) (2009) 011404

[17] E. Goulielmakis, M. Schultze, M. Hofstetter, V. S. Yakovlev, J. Gagnon, M. Uiberacker, A. L. Aquila, E. M. Gullikson, D. T. Attwood, R. Kienberger, F. Krausz, U. Kleineberg, Single-cycle nonlinear optics, Science 320 (5883) (2008) 1614-1617.

[18] M. Lein, N. Hay, R. Velotta, J. P. Marangos, P. L. Knight, Role of the intramolecular phase in high-harmonic generation, Phys. Rev. Lett. 88 (18) (2002) 183903

[19] T. Kanai, S. Minemoto, H. Sakai, Quantum interference during high-order harmonic generation from aligned molecules, Nature 435 (7041) (2005) 470-474.

[20] C. Vozzi, F. Calegari, E. Benedetti, J. P. Caumes, G. Sansone, S. Stagira, M. Nisoli, R. Torres, E. Heesel, N. Kajumba, J. P. Marangos, C. Altucci, R. Velotta, Controlling two-center interference in molecular high harmonic generation, Phys. Rev. Lett. 95 (15) (2005) 153902-153904.

[21] W. Boutu, S. Haessler, H. Merdji, P. Breger, G. Waters, M. Stankiewicz, L. Frasinski, R. Taïeb, J. Caillat, A. Maquet, P. Monchicourt, B. Carré, P. Salières, Coherent control of attosecond emission from aligned molecules, Nature Physics 4 (7) (2008) 545-549.

[22] J. Levesque, Y. Mairesse, N. Dudovich, H. Pepin, J. Kieffer, P. B. Corkum, D. M. Villeneuve, Polarization state of highorder harmonic emission from aligned molecules, Phys. Rev. Lett. 99 (24) (2007) 243001.

[23] R. R. Lucchese, G. Raseev, V. McKoy, Studies of differential and total photoionization cross sections of molecular nitrogen, Phys. Rev. A 28 (1982) 25722587.

[24] Y. Mairesse, S. Haessler, B. Fabre, J. Higuet,W. Boutu, P. Breger, E. Constant, D. Descamps, E. Mével, S. Petit, P. Salieres, Polarization-resolved pumpprobe spectroscopy with high harmonics, New Journal of Physics 10 (2008) 025028.

[25] E. Hijano, C. Serrat, G. Gibson, J. Biegert, Orbital geometry determined by orthogonal high-order harmonic polarization components, Phys. Rev. A 81 (2010) 041401.

[26] D. Shafir, Y. Mairesse, D. M. Villeneuve, P. B. Corkum, N. Dudovich, Atomic wavefunctions probed through strongfield light-matter interaction, Nature Phys. 5 (6) (2009) 412416.

[27] J. Itatani, D. Zeidler, J. Levesque, M. Spanner, D. M. Villeneuve, P. B. Corkum, Controlling high harmonic generation with molecular wave packets, Phys. Rev. Lett. 94 (12) (2005) 123902.

[28] S. Baker, J. S. Robinson, C. A. Haworth, H. Teng, R. A. Smith, C. C. Chirilă, M. Lein, J. W. G. Tisch, J. P. Marangos, Probing proton dynamics in molecules on an attosecond time scale, Science 312 (5772) (2006) 424-427.

[29] S. Haessler, W. Boutu, M. Stankiewicz, L. J. Frasinski, S. Weber, J. Caillat, R. Taieb, A. Maquet, P. Breger, P. Monchicourt, B. Carre, P. Salieres, Attosecond chirp-encoded dynamics of light nuclei, J. Phys. B 42 (13) (2009) 134002.

[30] J. P. Farrell, S. Petretti, J. Forster, B. K. McFarland, L. S. Spector, Y. V. Vanne, P. Decleva, P. H. Bucksbaum, A. Saenz, M. Guhr, Strong Field Ionization to Multiple Electronic States in Water, Phys. Rev. Lett. 107 (8) (2011) 083001.

[31] N. L. Wagner, A. Wuest, I. P. Christov, T. Popmintchev, X. Zhou, M. M. Murnane, H. C. Kapteyn, Inaugural article: Monitoring molecular dynamics using coherent electrons from high harmonic generation, Proc. Natl. Acad. Sci. USA 103 (36) (2006) 13279-13285.

[32] W. Li, X. Zhou, R. Lock, S. Patchkovskii, A. Stolow, H. C. Kapteyn, M. M. Murnane, Time-resolved dynamics in $\mathrm{N}_{2} \mathrm{O}_{4}$ probed using high harmonic generation, Science 322 (5905) (2008) 1207-1211.
[33] O. Smirnova, Y. Mairesse, S. Patchkovskii, N. Dudovich, D. Villeneuve, P. Corkum, M. Y. Ivanov, High harmonic interferometry of multi-electron dynamics in molecules, Nature 460 (7258) (2009) 972-977.

[34] S. Haessler, J. Caillat, W. Boutu, C. Giovanetti-Teixeira, T. Ruchon, T. Auguste, Z. Diveki, P. Breger, A. Maquet, B. Carre, R. Taieb, P. Salieres, Attosecond imaging of molecular electronic wave-packets, Nat Phys 6 (3) (2010) 200-206.

[35] A. D. Shiner, B. E. Schmidt, C. Trallero-Herrero, H. J. Wörner, S. Patchkovskii, P. B. Corkum, J.-C. Kieffer, F. Légaré, D. M. Villeneuve, Probing collective multi-electron dynamics in xenon with high-harmonic spectroscopy, Nature Physics 7 (2011) 464467.

[36] H. J. Worner, J. B. Bertrand, D. V. Kartashov, P. B. Corkum, D. M. Villeneuve, Following a chemical reaction using highharmonic interferometry, Nature 466 (7306) (2010) 604-607.

[37] H. J. Worner, J. B. Bertrand, B. Fabre, J. Higuet, H. Ruf, A. Dubrouil, S. Patchkovskii, M. Spanner, Y. Mairesse, V. Blanchet, E. Mevel, E. Constant, P. B. Corkum, D. M. Villeneuve, Conical intersection dynamics in $\mathrm{NO}_{2}$ probed by homodyne high-harmonic spectroscopy, Science 334 (6053) (2011) 208-212.

[38] J. Itatani, J. Levesque, D. Zeidler, H. Niikura, H. Pepin, J. C. Kieffer, P. B. Corkum, D. M. Villeneuve, Tomographic imaging of molecular orbitals, Nature 432 (7019) (2004) 867-871.

[39] B. K. McFarland, J. P. Farrell, P. H. Bucksbaum, M. Gühr, High harmonic generation from multiple orbitals in $\mathrm{N}_{2}$, Science 322 (5905) (2008) 1232-1235.

[40] O. Smirnova, S. Patchkovskii, Y. Mairesse, N. Dudovich, M. Y. Ivanov, Strong-field control and spectroscopy of attosecond electron-hole dynamics in molecules, Proc. Natl. Acad. Sci. USA 106 (39) (2009) 16556-16561.

[41] Z. Diveki, R. Guichard, A. Camper, J. Caillat, S. Haessler, T. Ruchon, T. Auguste, A. Maquet, B. Carré, R. Taieb, P. Salières, Spectrally-resolved multi-channel contributions to the harmonic emission in $\mathrm{N}_{2}$, New Journal of Physics 14 (2012) 023062.

[42] P. Salières, B. Carré, L. Le Déroff, F. Grasbon, G.G. Paulus, H. Walther, R. Kopold, W. Becker, D.B. Milosevic, A. Sanpera, M. Lewenstein, Science 292 (5518) (2001) 902-905.

[43] S. Haessler, J. Caillat, P. Salières, Self-probing of molecules with high harmonic generation, Journal of Physics B: Atomic, Molecular and Optical Physics 44 (20) (2011) 203001.

[44] J. Baggesen, L. Madsen, On the dipole, velocity and acceleration forms in high-order harmonic generation from a single atom or molecule, J. Phys. B 44 (2011) 115601.

[45] Y.-C. Han, L. B. Madsen, Comparison between length and velocity gauges in quantum simulations of high-order harmonic generation, Phys. Rev. A 81 (2010) 063430.

[46] M. Y. Ivanov, T. Brabec, N. Burnett, Coulomb corrections and polarization effects in high-intensity high-harmonic emission, Phys. Rev. A 54 (1) (1996) 742-745.

[47] E. V. Zwan, M. Lein, Control of recollision wave packets for molecular orbital tomography using short laser pulses, J. Phys. B 41 (7) (2008) 074009.

[48] A. T. Le, Della, P. Fainstein, D. Telnov, M. Lein, C. Lin, Theory of high-order harmonic generation from molecules by intense laser pulses, J. Phys. B 41 (8) (2008) 081002.

[49] M. V. Frolov, N. L. Manakov, T. S. Sarantseva, A. F. Starace, Analytic confirmation that the factorized formula for harmonic generation involves the exact photorecombination cross section, Phys. Rev. A 83 (2011) 043416.

[50] R. Murray, M. Spanner, S. Patchkovskii, M. Y. Ivanov, Tunnel ionization of molecules and orbital imaging, Phys. Rev. Lett. 106 (2011) 173001 
[51] M. V. Ammosov, N. B. Delone, V. P. Krainov, Tunnel ionization of complex atoms and of atomic ions in an alternating electromagnetic field, Sov. Phys. - JETP 64 (6) (1986) 1191-1194.

[52] A. T. Le, R. R. Lucchese, S. Tonzani, T. Morishita, C. D. Lin, Quantitative rescattering theory for high-order harmonic generation from molecules, Phys. Rev. A 80 (1) (2009) 013401.

[53] X. M. Tong, Z. X. Zhao, C. D. Lin, Theory of molecular tunneling ionization, Phys. Rev. A 66 (2002) 033402.

[54] D. B. Milošević, Strong-field approximation for ionization of a diatomic molecule by a strong laser field, Phys. Rev. A 74 (2006) 063404

[55] I. V. Litvinyuk, K. F. Lee, P. W. Dooley, D. M. Rayner, D. M. Villeneuve, P. B. Corkum, Alignment-dependent strong field ionization of molecules, Phys. Rev. Lett. 90 (23) (2003) 233003.

[56] T. Seideman, Revival structure of aligned rotational wave packets, Phys. Rev. Lett. 83 (24) (1999) 4971-4974.

[57] H. Stapelfeldt, T. Seideman, Colloquium: Aligning molecules with strong laser pulses, Rev. Mod. Phys. 75 (2) (2003) 543.

[58] F. Rosca-Pruna, M. J. J. Vrakking, Experimental observation of revival structures in picosecond laser-induced alignment of i2, Phys. Rev. Lett. 87 (15) (2001) 153902.

[59] N. Wagner, X. Zhou, R. Lock, W. Li, A. Wuest, M. Murnane, H. Kapteyn, Extracting the phase of high-order harmonic emission from a molecule using transient alignment in mixed samples, Phys. Rev. A 76 (6) (2007) 061403.

[60] C. Vozzi, M. Negro, F. Calegari, G. Sansone, M. Nisoli, S. De Silvestri, S. Stagira, Generalized molecular orbital tomography, Nature Physics 7 (10) (2011) 822-826.

[61] Z. Diveki, R. Guichard, T. Ruchon, A. Camper, D. Guénot, S. Haessler, J. Caillat, E. English, T. Auguste, P. Breger, A. Maquet, B. Carré, R. Taieb, P. Salières, to be published.

[62] E. V. van der Zwan, C. C. Chirilă, M. Lein, Molecular orbital tomography using short laser pulses, Phys. Rev. A 78 (3) (2008) 033410.

[63] M. Lein, Molecular imaging using recolliding electrons, Journal of Physics B: Atomic, Molecular and Optical Physics 40 (16) (2007) R135.

[64] V.-H. Le, A.-T. Le, R.-H. Xie, C. D. Lin, Theoretical analysis of dynamic chemical imaging with lasers using high-order harmonic generation, Phys. Rev. A 76 (1) (2007) 013413.

[65] Z. B. Walters, S. Tonzani, C. H. Greene, Limits of the plane wave approximation in the measurement of molecular properties, J. Phys. Chem. A 112 (39) (2008) 9439-9447.

[66] J. Muth-Böhm, A. Becker, F. H. M. Faisal, Suppressed molecular ionization for a class of diatomics in intense femtosecond laser fields, Phys. Rev. Lett. 85 (2000) 2280-2283.

[67] Y. Mairesse, J. Higuet, N. Dudovich, D. Shafir, B. Fabre, E. Mével, E. Constant, S. Patchkovskii, Z. Walters, M. Y. Ivanov, O. Smirnova, High harmonic spectroscopy of multichannel dynamics in strong-field ionization, Phys. Rev. Lett. 104 (2010) 213601.

[68] Z. B. Walters, O. Smirnova, Attosecond correlation dynamics during electron tunnelling from molecules, Journal of Physics B: Atomic, Molecular and Optical Physics 43 (16) (2010) 161002.

[69] T. Kanai, E. J. Takahashi, Y. Nabekawa, K. Midorikawa, Destructive interference during high harmonic generation in mixed gases, Phys. Rev. Lett. 98 (15) (2007) 153904.

[70] R. Torres, T. Siegel, L. Brugnera, I. Procino, J. G. Underwood, C. Altucci, R. Velotta, E. Springate, C. Froud, I. C. E. Turcu, S. Patchkovskii, M. Y. Ivanov, O. Smirnova, J. P. Marangos, Revealing molecular structure and dynamics through high-order harmonic generation driven by mid-ir fields, Phys. Rev. A 81 (2010) 051802 .

[71] B. K. McFarland, J. P. Farrell, P. H. Bucksbaum, M. Gühr, Highorder harmonic phase in molecular nitrogen, Phys. Rev. A 80
(2009) 033412

[72] H. J. Wörner, J. B. Bertrand, P. Hockett, P. B. Corkum, D. M. Villeneuve, Controlling the interference of multiple molecular orbitals in high-harmonic generation, Phys. Rev. Lett. 104 (2010) 233904

[73] Y. Mairesse, J. Levesque, N. Dudovich, P. Corkum, D. Villeneuve, High harmonic generation from aligned moleculesamplitude and polarization, Journal of Modern Optics 55 (16) (2008) 2591-2602.

[74] J. Farrell, B. K. McFarland, M. Gühr, P. Bucksbaum, Relation of high harmonic spectra to electronic structure in $\mathrm{N}_{2}$, Chemical Physics 366 (2009) 15-21.

[75] C. Jin, A.-T. Le, C. D. Lin, Medium propagation effects in highorder harmonic generation of Ar and $\mathrm{N}_{2}$, Phys. Rev. A 83 (2) (2011) 023411

[76] A. Harvey, An attosecond window into the life of a shape resonance, Poster at Advances in Strong-Field and Attosecond Physics at University College, London (2010).

[77] S. Sukiasyan, S. Patchkovskii, O. Smirnova, T. Brabec, M. Y. Ivanov, Exchange and polarization effect in high-order harmonic imaging of molecular structures, Phys. Rev. A 82 (2010) 043414.

[78] Y. Mairesse, A. de Bohan, L. Frasinski, H. Merdji, L. Dinu, P. Monchicourt, P. Breger, M. Kovacev, R. Taïeb, B. Carré, H. Muller, P. Agostini, P. Salières, Attosecond synchronization of high-harmonic soft X-rays, Science 302 (5650) (2003) 15401543.

[79] Y. Mairesse, private communication.

[80] J. Tate, T. Auguste, H. G. Muller, P. Salières, P. Agostini, L. F DiMauro, Scaling of wave-packet dynamics in an intense midinfrared field, Phys. Rev. Lett. 98 (1) (2007) 013901.

[81] T. Popmintchev, M.-C. Chen, A. Bahabad, M. Gerrity, P. Sidorenko, O. Cohen, I. Christov, M. Murnane, H. Kapteyn, Phase matching of high harmonic generation in the soft and hard $\mathrm{X}$-ray regions of the spectrum, Proc. Natl Acad. Sci. USA 106 (2009) 1051610521.

[82] C. Jin, J. B. Bertrand, R. R. Lucchese, H.J. Wörner, P.B. Corkum, D. M. Villeneuve, A.-T. Le, C. D. Lin, Intensity dependence of multiple orbital contributions and shape resonance in high-order harmonic generation of aligned $\mathrm{N}_{2}$ molecules, Phys. Rev. A 1 (2012) 013405. 